\title{
Malignant Migrating Partial Seizures of Infancy (MMPSI): Expansion of Clinical Phenotypic and Genotypic Spectra
}

\author{
liwen wu \\ Hunan Children's Hospital \\ Fang Cai \\ Chenzhou No 1 People's Hospital \\ Siyi Gan \\ Hunan Children's Hospital \\ Sai Yang \\ Hunan Children's Hospital \\ Xiaofan Yang \\ Qilu Hospital of Shandong University Qingdao \\ haiyan Yang ( $D$ 871562488@qq.com) \\ Hunan Children's Hospital https://orcid.org/0000-0003-1164-8788
}

\section{Research Article}

Keywords: Malignant migrating partial seizures of infancy, migrating seizures, genetics, Developmental and Epileptic Encephalopathy

Posted Date: September 2nd, 2021

DOI: https://doi.org/10.21203/rs.3.rs-856006/v1

License: (c) (i) This work is licensed under a Creative Commons Attribution 4.0 International License. Read Full License 


\section{Abstract}

Background

MMPSI is a rare early infantile epileptic encephalopathy with unknown etiology and poor prognosis. With the development of genetic techniques, an increasing number of novel pathogenic genes have been shown to be related to MMSPI. This study included 36 patients who were diagnosed with MMPSI; the clinical features, etiology, treatment strategies and outcomes of these patients were determined to explore new genetic etiology and new precision medicine treatment strategies.

Results

36 patients were enrolled. The main seizure types were focal, tonic and spasms. The main EEG findings were seizure migration and hypsarrhythmia. $17 / 36$ cases had causative variants across 11 genes, including 6 novel MMPSI genes: PCDH19, ALDH7A1, DOCK6, PRRT2, ALG1 and ATP7A. 13/36 patients had ineffective seizure control, $14 / 36$ patients had severe retardation and $6 / 36$ patients died. Of them, the genes for ineffective seizure control, severe retardation or death include $K C N T 1, S C N 2 A, S C N 1 A, A L G 1, A T P 7 A$ and WWOX. Genes associated with seizure-free, mild-moderate retardation or normal development included PRRT2, SCN2A, ALDH7A1 and PNPO. Vitamin B6 allowed patients with ALDH7A1 and PNPO mutations to achieve seizure-free status, oxcarbazepine was effective for patients with SCN2A, ATP7A, WWOX, and PRRT2 mutations, and ACTH was partly effective for DOCK6 mutation patients with spasms and hypsarrhythmia. 17 patients had abnormal MRI, of which 8 had ineffective seizure control, 7 had severe retardation and 4 (4/17, 23.5\%) died. 13 patients had hypsarrhythmia, of which 6 had ineffective seizure control, 6 had severe retardation and 2 died. Also, 7 patients had burst suppression, of which 1 had ineffective seizure control, 3 had severe retardation and 3 died.

Conclusion

This study is the first to report that $A L D H 7 A 1, A T P 7 A, D O C K 6, P R R T 2, A L G 1$, and PCDH19 mutations cause the phenotypic spectrum of MMPSI to expand the genotypic spectrum. The genes KCNT1, SCN2A, SCN1A, ALG1, ATP7A and WWOX may be associated with poor prognosis. The patients presenting with MRI abnormalities, hypsarrhythmia and burst suppression in EEG may be associated with poor prognosis. Through early diagnosis with genetic tests and the administration of the corresponding precise treatment, the outcomes of MMPSI can be notably improved.

\section{Background}

Malignant migrating partial seizures of infancy (MMPSI) syndrome, which was first reported in 1995 [1], is a rare early infantile epileptic encephalopathy with poor prognosis, presenting with focal seizures in the first year of life. The main clinical features are seizure onset in the first 6 months of life, occurrence of almost continuous migrating polymorphous focal seizures, combined with multifocal ictal electroencephalography (EEG) discharges, and progressive deterioration of psychomotor development [2].

Previously, MMPSI was reported as an epilepsy syndrome with unknown etiology and poor prognosis [3]. Currently, in pace with the continuous development of genetic techniques, an increasing number of novel pathogenic genes have been shown to be related to MMSPI, including GABRA1, GABRB1, GABRB3, KCNQ2, BRAT1, HCN1, SCN8A, SMC1A, TBC1D24, QARS, ATP1A3, CDKL5, PIGA, ITPA, AIMP1, KARS, WWOX, KCNT1, TBC1D24, SLC25A22, SCN1A, SCN2A, SLC12A5, PLCB1 and PNPO [2, 4-6]. Correspondingly, precision medicine treatments were adopted and partly improved patient outcomes. This study included 36 patients who were diagnosed with MMPSl; the clinical features, etiology, treatment strategies and outcomes of these patients were determined to explore new genetic etiology and new precision medicine treatment strategies.

\section{Results}

\section{Clinical phenotypic spectrum}

A total of 36 patients were enrolled in this study, and all patients fulfilled the inclusion criteria. We presented the phenotypic summary of the cohort (Table 1 ). Among them, 25 (69.4\%) were male, and 11 (30.6\%) were female. Eleven patients (30.6\%) had seizure onset at 4 hours to 3 days after birth. Ten (27.8\%) patients had a family history of epilepsy, febrile seizure or mental retardation, or stillbirth. There were 10 (27.8\%) patients with congenital heart disease, 8 (22.2\%) patients with perinatal history, 1 (2.8\%) patients with intracranial hemorrhage, $1(2.8 \%)$ patients with inguinal hernia, $3(8.3 \%)$ patient with intracranial infection, 1 (2.8\%) patient with Menkes disease and 1 (2.8\%) patient with congenital disorder of glycosylation, type lk.

Seizure type at onset included focal seizure (36/36, $100 \%)$; tonic seizure (21/36, $58.3 \%)$; spasms (8/36, $22.2 \%)$; clonic seizure (5/36, $13.9 \%)$ and tonic-clonic, autonomic seizures and others (8/36, 22.2\%). All the patients had seizure migration; hypsarrhythmia (classic or modified) was seen in 13 (13/36, $36.1 \%$ ) patients, and a burst-suppression pattern was observed in $7(7 / 36,19.4 \%)$ patients. All patients underwent brain MRI and/or CT examinations, 17 of whom had abnormal brain imaging findings. Diffuse cerebral atrophy (with an increase in extra axial fluid) was observed in 3 children. Four cases demonstrated forehead dysplasia or widened brain space, 3 presented with hippocampal sclerosis, 3 presented with brain softening foci, 1 presented with dysplasia of the corpus callosum, 5 presented with subependymal cyst, 1 presented with pachygyria and 1 presented with meningeal reinforcement. Specifically, one of the 3 children with cerebral atrophy was accompanied with hippocampal sclerosis. One child had brain softening foci, hippocampal sclerosis, subependymal cyst and widened brain space.

The etiological composition of the 36 patients in this cohort was determined by the International League Against Epilepsy (ILAE) 2017 etiological classification criteria. Accordingly, there were 17 (17/36, 47.2\%) cases of inheritance, of which $6(6 / 17,35.3 \%)$ had severe retardation, $3(3 / 17,17.6 \%)$ died and $2(2 / 17,11.8 \%)$ was normal. There were $17(17 / 36,47.2 \%)$ cases of structure (8 cases were inherited), of which 7 ( $7 / 17,41.2 \%)$ had severe retardation, 4

Page 2/13 
$(4 / 17,23.5 \%)$ died and $1(1 / 17,5.9 \%)$ was normal. Three patient $(3 / 36,8.3 \%)$ had infectivity indications, and 1 died. There were two $(2 / 36,5.6 \%)$ cases of metabolic syndrome (both cases were inherited), of which 1 had severe retardation and 1 died. There was no immunity and 10 (10/36, 27.8\%) cases for unknown reasons, of which $5(5 / 10,50.0 \%)$ had severe retardation and $1(1 / 10,10.0 \%)$ were normal.

\section{Genotypic spectrum}

There were $26(26 / 36,72.2 \%)$ patients who underwent genetic testing, including $15(15 / 26,57.7 \%)$ with whole exome sequencing (WES), 11 (11/26, $42.3 \%)$ with an epilepsy gene panel, $3(3 / 26,11.5 \%)$ with mitochondrial genome sequencing, $1(1 / 26,3.8 \%)$ with chromosome karyotype analysis and 1 (1/26, $3.8 \%)$ with copy number variant (CNV). Specifically, one of the 2 patients with mitochondrial genome sequencing and WES, one of the 1 patient with mitochondrial genome sequencing, WES, chromosome karyotype analysis and CNV. Seventeen (17/36, 47.2\%) cases had causative variants across 11 genes (Table 2$)$, including 6 novel MMPSI genes: de novol PCDH19; paternal ALDH7A1, DOCK6, PRRT2 and ALG1; maternal ALDH7A1, ATP7A, DOCK6 and ALG1. Five genes have been reported in previous literature, including de novo KCNT1, SCN2A, SCN1A, paternal PNPO, WWOX, maternal PNPO and WWOX. The most frequently implicated genes were $\operatorname{KCNT1}(3 / 36,8.3 \%)$ and $\operatorname{SCN} 2 A(6 / 36,16.7 \%)$.

\section{MMPSI phenotype-genotype correlation}

Phenotypic data are summarized according to each gene in Table 3. We highlight the phenotypes of the five most frequently implicated genes for MMPSI here.

KCNT1 MMPSI patients $(\mathrm{n}=3)$

Three patients ( 3 males) with KCNT1 variants aged 1 year and 4 months to 2 years were studied (median 1 year 4 months). The median seizure onset was 1 months (range 15 days to 4 months). All had focal seizures, 1 had tonic seizures, 2 had spasms seizures, and 2 had autonomic seizures. All had normal brain imaging findings. EEG showed seizure migration in all patients, hypsarrhythmia in $2 / 3$, and burst suppression in $1 / 3$. Two patients had severe developmental retardation, and one died. The age of death was 2 years.

\section{SCN2A MMPSI ( $\mathrm{n}=6)$}

There were 6 patients with SCN2A MMPSI studied at 1 month to 6 years of age (median 1 years 5 months) with median seizure onset at 2 days (range 1 day to 9 months). All had focal seizures, 4 had tonic seizures, 2 had spasms seizures, and 1 had an autonomic seizure. A total of $2 / 6$ had normal brain imaging findings, and $4 / 6$ had abnormal imaging findings. EEG data showed seizure migration in all patients, hypsarrhythmia in $3 / 6$, and burst suppression in $2 / 6$. The effective drugs for 6 patients with seizure control were oxcarbazepine, carbamazepine, vigabatrin and levetiracetam. Three patients had severe developmental retardation, three patients had mild-moderate development retardation, and no patients died.

PNPO and ALDH7A1 MMPSI (n=2)

One patient (female) with PNPO variants was studied at 4 years and 4 months of age. Seizure onset was 2 days after birth. She had focal seizures and abnormal brain imaging findings. One patient (male) with ALDH7A1 variants was studied at 3 years and 10 months of age. Seizure onset was 1 month. He had focal and clonic seizures and normal brain imaging. There was no hypsarrhythmia or burst suppression in the EEG of these two patients. Vitamin B6 was effective in both patients. One patient had only mild development retardation, and one patient had normal development.

PRRT2 MMPSI ( $\mathrm{n}=1)$

One patient (female) with PRRT2 variants was studied at 10.5 months of age. Seizure onset was 3 months. She had focal seizures and normal brain imaging findings. No hypsarrhythmia or burst suppression was evident in the EEG data of the patient. Oxcarbazepine was effective in controlling seizures in this patient, who had normal development.

\section{Treatment}

Antiepileptic treatment effects on seizures were analyzed in 36 patients with MMPSI (Table 3). In addition to classical antiepileptic drugs (AEDs), vitamin B6 and adrenocorticotropic hormones (ACTH) were used to treat MMPSI patients. Vitamin B6 allowed patients with ALDH7A1 and PNPO mutations to achieve seizure-free status, oxcarbazepine was effective for patients with SCN2A, ATP7A, WWOX, and PRRT2 mutations, and ACTH was partly effective for DOCK6 mutation patients with spasms and hypsarrhythmia.

\section{Outcome}

We obtained data regarding seizure control and motor development for 36 patients: 14/36 (38.9\%) patients were seizure free, and $9 / 36$ (25.0\%) patients had seizures reduced $>50 \%$. However, $13 / 36$ (36.1\%) patients had ineffective seizure control. 14/36 (38.9\%) patients had severe retardation, $13 / 36$ (36.1\%) patients had mild-moderate retardation, and 3/36 (8.3\%) patients had normal development (Table 3). Our cohort had a high mortality rate: $6 / 36$ (16.7\%) patients died at a median age of 8 months (range 3 months to 2 years). In $3 / 6$ patients who died, pathogenic variants were identified in the following genes: $K C N T 1$ (1), SCN1A (1) and $A L G 1$ (1), with 3 cases remaining unsolved. The genes for ineffective seizure control and severe retardation include $K C N T 1$ (2), SCN2A (1), ATP7A (1) and WWOX (1). Genes associated with seizure-free, mild-moderate retardation or normal of mental and motor development included PRRT2 (1), SCN2A (3), ALDH7A1 (1) and PNPO (1).

Of the 36 patients with MMPSI, 19 had normal MRI findings, of which $9(9 / 19,47.4 \%)$ were seizure free, $9(9 / 19,47.4 \%)$ had ineffective seizure control, 7 $(7 / 19,36.8 \%)$ had severe retardation and $2(2 / 19,10.5 \%)$ died. Also, 17 had abnormal MRI, of which $5(5 / 17,29.4 \%)$ were seizure free, $8(8 / 17,47.1 \%)$ had ineffective seizure control, $7(7 / 17,41.2 \%)$ had severe retardation and $4(4 / 17,23.5 \%)$ died. The details are shown in Table 4. 
Of the 36 patients with MMPSI, 13 had hypsarrhythmia, of which $5(5 / 13,38.5 \%)$ were seizure free, $6(6 / 13,46.1 \%)$ had ineffective seizure control, $6(6 / 13$, $46.1 \%)$ had severe retardation, $2(2 / 13,15.4 \%)$ died and $0(0 / 13,0 \%)$ were normal; also, 7 had burst suppression, of which $1(1 / 7,14.3 \%)$ were seizure free, 1 $(1 / 7,14.3 \%)$ had ineffective seizure control, $3(3 / 7,42.9 \%)$ had severe retardation, $3(3 / 7,42.9 \%)$ died and $0(0 / 3,0 \%)$ were normal. 3 patients had hypsarrhythmia and burst suppression, of which $1(1 / 3,33.3 \%)$ were seizure free, $1(1 / 3,33.3 \%)$ had ineffective seizure control, $2(2 / 3,66.7 \%)$ had severe retardation, $0(0 / 3,0 \%)$ died and $0(0 / 3,0 \%)$ were normal, and 19 had no hypsarrhythmia or burst suppression, of which $8(8 / 19,42.1 \%)$ were seizure free, 7 $(7 / 19,36.8 \%)$ had ineffective seizure control, $5(5 / 19,26.3 \%)$ had severe retardation, $2(2 / 19,10.5 \%)$ died and $3(3 / 19,15.8 \%)$ were normal. The details are shown in Table 5.

\section{Discussion}

MMPSI is characterized by nearly continuous seizures involving multiple independent areas of both hemispheres with arrested psychomotor development. It is an age-dependent, often overlooked syndrome among the epileptic encephalopathies that can occur within the first 6 months of life [7]. It may therefore be included among epileptic encephalopathies, together with others beginning in the same period, such as early infantile epileptic encephalopathy, early myoclonic encephalopathy, and infantile spasms [8]. The clinical manifestations of children with MMPSI are characterized by migrating and focal seizures, with an onset peak from 40 days to 3 months. Symptoms can manifest on EEG readings as diffuse slow waves in the background, multifocal discharge with or without spastic between episodes, typical or atypical hypsarrhythmia and never a suppression burst pattern [3]. MMPSI has no specific neuroimaging changes, and the neuroimaging abnormalities reported in the current literature include delayed myelination, abnormal signals in the basal ganglia, dysplasia in the corpus callosum, diffuse brain atrophy, microcephaly, multiple cortical developmental malformations with multiple cerebellar gyri, focal cortical dysplasia, and hippocampal sclerosis $[2,8,9]$. We described the phenotype spectrum of 36 patients with MMPSI in this study. All patients had clinical seizure migration associated with a significant impact on development. We identified several key phenotypic features that have only been rarely reported in the MMPSI phenotypic spectrum: 11 (11/36, 30.6\%) patients had suspected in utero seizures with postnatal seizures described between 4 hours and 3 days, 8 (8/36, $22.2 \%)$ patients had epileptic spasms, 7 (7/36, 19.4\%) had a burst-suppression pattern in EEG activity, 1 patient had congenital disorder of glycosylation-type Ik, 1 patient had Menkes disease, 3 patients had intracranial infection, 1 patient had inguinal hernia, 1 patient had intracranial hemorrhage, 1 patient had premature disease, 10 patients had congenital heart disease (open foramen ovale, patient ductus arteriosus), and 8 patients had a perinatal history (pneumonia, HIE, intrauterine distress). Furthermore, it is mostly believed that the etiology of MMPSI is caused by genetic mutations [7]. According to our etiological analysis, the genetic etiology accounted for 17/36 cases, and brain injury accounted for 17/36 cases, including 1 case of MMPSI caused by hypoxic-ischemic encephalopathy (HIE); hence, HIE sequelae can also lead to MMPSI. Analysis of etiology and prognosis results suggests that structural and infectious causes may be related to poor prognosis.

We describe the genotypic spectrum of 17 patients with MMPSI. We highlight the extensive genetic heterogeneity of MMPSI, which is similar to that in other epilepsy syndromes, such as infantile spasms (West syndrome) and Lennox-Gastaut syndrome, but with a considerably higher yield on current testing. We identified the etiology in $47.2 \%$ of our cohort, including 6 novel MMPSI genes. The most commonly involved genes were $K C N T 1$ (8.3\%) and SCN2A (16.7\%), together explaining $25.0 \%$ of MMPSI cases. Among the 3 patients with $K C N T 1$ gene mutations, the mutation sites of 1 patients were classified as a variant of unknown significance (VUS) by the American College of Medical Genetics (ACMG), and among the 6 patients with SCN2A gene mutations, the mutation site of 1 patient was classified as a VUS by the ACMG. However, according to the analysis of the clinical phenotypes and responses to drug therapy of the patients, we still believed that $K C N T 1$ and $S C N 2 A$ were the pathogenic genes of patients. This pattern differs from that reported in patients with neonatal-onset developmental and epileptic encephalopathy [10], including Ohtahara syndrome [11], for which KCNQ2 and SCN2A are most common. These studies highlight the importance of classifying a patient's epilepsy syndrome, which influences genetic testing and interpretation [12]. Epileptic spasms have only been rarely reported in MMPSI patients [2]. In this series, 8/36 patients (22.2\%) had epileptic spasms, including those with KCNT1, SCN2A, SCN1A, DOCK6 and PCDH19 variants. Epileptic spasms are a hallmark of $C D K L 5$ encephalopathy, but MMPSI had not been previously described in this disease. Whether other genes predispose patients with MMPSI to epileptic spasms will require studies with large cohorts to enable phenotype-genotype correlation.

The PNPO gene encodes pyridoxine 5-prime-phosphate oxidase, and the maintenance of optimal pyridoxal 5-prime-phosphate levels in the brain is important in many neurologic disorders in which neurotransmitter metabolism is disturbed, which is associated with autosomal recessive pyridoxamine 5 '-phosphate oxidase deficiency [13]. The PNPO gene was MMPSI-related genes reported in previous literature. In our study cohort, although the mutation sites of the gene were classified as VUS by the ACMG, taking into account the analysis of the clinical phenotypes and responses to drug therapy of the patient, we still believed that $P N P O$ was the pathogenic genes of patients.

We discovered 6 novel MMPSI genes in our cohort - de novo PCDH19; paternal ALDH7A1, DOCK6, PRRT2 and ALG1; and maternal ALDH7A1, ATP7A, DOCK6 and ALG1 - encoding a wide range of proteins. These genes have not been described in patients with MMPSI, but they have been associated with other neurological diseases.

Considering the $P C D H 19$ gene first, most of the $P C D H 19$ gene variants resulted in protein termination and nonsense-mediated decay and affected $P C D H 19$ through impaired calcium binding, which is associated with X-linked early infantile epileptic encephalopathy-9 (EIEE-9) in females [14]. Considering that one of our patients was a female and had a de novo mutation of the $P C D H 19$ gene, which is consistent with the X-linked genetic classification and the common clinical phenotype of spasms seizure and delayed motor development, the software predicted the likely pathogenicity of the mutation; thus, these findings suggest that the $\mathrm{PCDH} 19$ gene could be associated with MMPSI.

The ALDH7A1 gene encodes an aldehyde dehydrogenase that is an alpha-aminoadipic semialdehyde dehydrogenase in the pipecolic acid pathway of lysine catabolism, which is associated with autosomal recessive pyridoxine-dependent epilepsy [15]. Considering that some of our patients had paternal and maternal mutations of the $A L D H 7 A 1$ gene, which is consistent with the autosomal recessive genetic classification and the common clinical phenotype of 
seizures, delayed motor development and effective treatment with vitamin B6, the software predicted the likely pathogenicity of the mutation; thus, these findings suggest that the ALDH7A1 gene could be associated with MMPSI.

DOCK6 belongs to subfamily $\mathrm{C}$ of the DOCK family and has a role in remodeling the actin cytoskeleton by functioning as a GEF for both CDC42 and RAC1 [16], which is associated with autosomal recessive Adams-Oliver syndrome-2. Adams-Oliver syndrome is a multiple congenital anomaly syndrome that is characterized by aplasia cutis congenita (ACC) as well as terminal transverse limb defects (TTLD) in addition to variable involvement of the brain, eyes, and cardiovascular system [17]. Although one of our patients had maternal and paternal mutations of the DOCK6 gene, which is consistent with the autosomal recessive genetic classification, and the software predicted the likely pathogenicity of the mutation, the patient lacked the typical clinical phenotype of AdamsOliver syndrome-2. Therefore, we could not determine whether the DOCK6 gene was the causative gene of MMPSI, and further data are needed for verification.

The PRRT2 gene encodes proline transmembrane protein 2 and is involved in signal transduction between neurons. PRRT2-associated paroxysmal movement disorders (PRRT2-PxMD) include autosomal dominant genetic paroxysmal kinesigenic dyskinesia (PKD), benign familial infantile epilepsy (BFIE), paroxysmal kinesigenic dyskinesia with infantile convulsions (PKD/IC), and hemiplegic migraine (HM). The characteristics of BFIE include early onset, cluster seizures that could be self-healing or that respond well to the treatment of sodium channel blockers, and normal development. In addition, PRRT2 pathogenic variants have been identified in other childhood-onset movement disorders and different types of seizures, suggesting that the understanding of the spectrum of PRRT2PxMD is still evolving [18]. The patient with this mutation in our cohort was very interesting. He presented with very frequent cluster seizures when he was 3 months old, video-EEG demonstrated migrating seizures and typical background characteristics of MMSPI, and he experienced obvious cognitive retardation after the onset of epilepsy. He achieved seizure-free status after the administration of oxcarbazepine, and follow-up showed normal cognitive development. The patient had a paternal mutation of the PRRT2 gene-his father had symptoms of dystonia in his youth-and the software predicted the likely pathogenicity of the mutation. We considered that the PRRT2 gene was the causative gene of MMPSI, and further data were needed for verification.

In the 10th patient, two pathogenic genes were detected simultaneously, namely, WWOX and ATP7A. The clinical phenotypes reported in the literature were Xlinked Menkes disease, occipital horn syndrome, distal spinal muscular atrophy 3, epileptic encephalopathy, early infantile 28 , esophageal squamous cell carcinoma, somatic and spinocerebellar ataxia, and autosomal recessive 12, which theoretically can cause epileptic encephalopathy [19-23]. This patient also had a significant decrease in blue copper protein, had hair and skin color changes and could have been diagnosed with Menkes disease. WWOX conformed to the genetic classification. One of the two mutation sites was a pathogenic mutation, and the other was a VUS. We could not confirm whether it was a copathogenic gene.

The ALG1 gene encodes mannosyltransferase I (MTI). The biosynthesis of lipid-linked oligosaccharides is highly conserved among eukaryotes and is catalyzed by 14 glycosyltransferases in an ordered stepwise manner. MT I catalyzes the first mannosylation step in this process [24]. The gene variants can result in autosomal recessive congenital disorder of glycosylation type $\mathrm{lk}[25,26]$. The characteristics of congenital disorder of glycosylation type $\mathrm{lk}$ includes feeding problems and diarrhea, profound hypoproteinemia with massive ascites, muscular hypertonia, seizures refractory to treatment, recurrent episodes of apnea, cardiac and hepatic involvement and coagulation anomalies [27]. The clinical characteristics of this patient were consistent with the above core symptoms, the genetic results were consistent with the law of autosomal recessive inheritance, and the software predicted the likely pathogenicity of the mutation. We considered the ALG1 gene to be a newly pathogenic gene of MMPSI.

Most patients with MMPSI are refractory to AEDs, but some have shown good progression or a near satisfactory response to treatment. The AEDs used alone or in combination with one another that may achieve seizure control or reduction are potassium bromide, levetiracetam, ACTH, stiripentol, clonazepam, and rufinamide [28]. Mikati et al. reported that quinidine is effective in the treatment of patients with KCNT1 gene mutations in MMPSI [29]. At present, there are few literature reports on the use of corresponding effective drug treatments for patients with different MMPSI gene mutations. In our cohort, we determined that vitamin B6 could allow patients with ALDH7A1 and PNPO mutations to achieve seizure-free status. Oxcarbazepine was effective for patients with SCN2A, ATP7A+WWOX, and PRRT2 mutations. One of the patients with a maternal SCN2A heterozygous mutation was treated with oxcarbazepine; subsequently, the patient's convulsions were controlled. The patient's mother had a normal heterozygote phenotype, which was consistent with the pathogenesis of autosomal dominant inheritance (incomplete penetrance). ACTH was partly effective for patients with DOCK6 mutations who had spasms and hypsarrhythmia.

While seizure outcomes and developmental prognoses are generally poor in MMPSI, there are rare reports of mildly affected patients [30]. In our study cohort, the incidence of poor prognosis was also relatively high; $6 / 36$ (16.7\%) patients died, and the related pathogenic genes were $K C N T 1, S C N 1 A, A L G 1$. $14 / 36$ (38.9\%) patients had severe retardation, and the genes for ineffective seizure control and severe retardation included KCNT1, SCN2A, WWOX and ATP7A. The results indicated that the related pathogenic genes $K C N T 1, S C N 1 A, A L G 1, S C N 2 A, W W O X$ and ATP7A may be associated with ineffective seizure control and poor prognoses. While all patients experienced refractory epilepsy early in the course of the disease, $3 / 36$ (8.3\%) patients had normal mental and motor development. Genes associated with seizure-free, mild-moderate retardation or normal of mental and motor development included PRRT2, SCN2A, ALDH7A1, $P C D H 19$ and $P N P O$.

In addition, we compared the association of MRI abnormalities, hypsarrhythmia and burst suppression in EEG with poor prognosis. The results found that patients with MMPSI characterized by abnormal MRI, hypsarrhythmia and burst suppression in EEG have a higher incidence of ineffective seizure control, severe retardation and a higher mortality rate. The results suggest that MMPSI patients who present with abnormal MRI, hypsarrhythmia and burst suppression in EEG may be associated with ineffective seizure control and poor prognosis. We need to further expand the sample to analyze and confirm these correlations.

\section{Conclusions}

In conclusion, the clinical phenotypic spectrum, such as seizure types and EEG findings, of MMPSI overlapped with infantile spasm and Ohtahara syndrome. MMPSI patients who present with abnormal MRI findings, hypsarrhythmia and burst suppression in EEG may be associated with ineffective seizure control 
and poor prognosis. Etiological analysis showed that in addition to genetic mutations, structural brain injury such as HIE could also be secondary to MMPSI, and structural and infectious etiology may be associated with poor prognosis. This study is the first to report that $A L D H 7 A 1, A T P 7 A, D O C K 6, P R R T 2$,

$A L G 1$, and PCDH19 mutations cause the phenotypic spectrum of MMPSI to expand the genotypic spectrum. The genes KCNT1, SCN1A, ALG1, SCN2A, WWOX and $A T P 7 A$ may be associated with ineffective seizure control and poor prognosis. Through early diagnosis with genetic tests and the administration of the corresponding precise treatment, the outcomes of MMPSI can be notably improved.

\section{Methods}

\section{Participants and phenotyping}

Our MMPSI cohort comprised 36 patients from Hunan Children's Hospital and Qilu Hospital of Shandong University. A multicenter retrospective case study was performed over a 10-year period (January 2010 to January 2020). The study was performed in accordance with the Declaration of Helsinki, with the approval of the study protocol by an independent ethics committee or institutional review board at each site. All patients provided written informed consent before participation.

Study inclusion criteria [6] were as follows: according to current literature reports, the maximum age of onset of children with MMPSI is 9 months, so we set the age of onset at 9 months; focal motor seizures at onset; multifocal seizures intractable to conventional antiepileptic drugs; initial EEG may be normal but show a subsequent development of characteristic changes, including interictal multifocal spikes and ictal independent, unilateral and migrating involvement of different cortical areas with clinical-EEG correlation; and delayed developmental progress or signs of psychomotor regression associated with seizure onset. The phenotypic information of all patients was collated on clinical presentation, disease course, EEG, neuroimaging, treatment strategies and the results of neurometabolic and diagnostic genetic investigations. All patients were followed up every 1-6 months by telephone or outpatient department.

\section{Genetic tests}

Genetic testing was carried out using chromosome karyotype analysis, CNV analysis, mitochondrial genome sequencing, epilepsy gene panels and WES. CNV analysis was performed with the Illumina HumanOmniZhonghua-8 Bead Chip; Mitochondrial genome sequencing was subsequently performed on an Illumina HiSeq 2000 platform (Illumina, San Diego, CA, USA); details were provided by our team previously[31]. The epilepsy gene panel contained 265 epilepsyassociated genes was performed on methods previously reported by Lemke et al [32]. WES was also performed based on methods previously reported by our team [31] by using Illumina HiSeq X Ten (Illumina, San Diego, CA, USA) with 150-bp paired-end reads.

\section{Abbreviations}

AEDs: antiepileptic drugs; ACTH: adrenocorticotropic hormones; ACC: aplasia cutis congenita; BFIE: benign familial infantile epilepsy; CNV: copy number variant; EEG: electroencephalography; EIEE-9: early infantile epileptic encephalopathy-9; HM: hemiplegic migraine; HIE: hypoxic-ischemic encephalopathy; ILAE: International League Against Epilepsy; IC: infantile convulsions; MMPSI: Malignant migrating partial seizures of infancy; MT I: mannosyltransferase I; PRRT2-PxMD: PRRT2-associated paroxysmal movement disorders; PKD: paroxysmal kinesigenic dyskinesia; TTLD: terminal transverse limb defects; VUS: variant of unknown significance; WES: whole exome sequencing

\section{Declarations}

\section{Acknowledgments}

We wish to thank the patients for the participation in this study.

\section{Authors' contributions}

LW conducted the literature review and drafted the manuscript. FC, SG and SY made substantial contributions to the conception and interpretation of data. XY and HY were responsible for revising the manuscript critically and gave final approval of the version to be published.

\section{Funding}

This work was supported by grants from the National Natural Science Foundation of China (No. 81671297).

\section{Data Availability Statement}

The datasets used and/or analyzed during the present study are available from the corresponding author on reasonable request.

\section{Ethics approval and consent to participate}

The study was performed in accordance with the Declaration of Helsinki, with the approval of the study protocol by an independent ethics committee or institutional review board at each site. All patients provided written informed consent before participation.

\section{Consent for publication}

All authors have approved the final article and its publication. 


\section{Conflict of interest}

The authors declare that they have no competing interests.

\section{References}

1 Coppola G, Plouin P, Chiron C, Robain O, Dulac O. Migrating partial seizures in infancy: a malignant disorder with developmental arrest. Epilepsia 1995; 36(10): 1017-1024.

2 McTague A, Appleton R, Avula S, Cross JH, King MD, Jacques TS, et al. Migrating partial seizures of infancy: expansion of the electroclinical, radiological and pathological disease spectrum. Brain 2013; 136(5): 1578-1591.

3 Coppola G. Malignant migrating partial seizures in infancy: an epilepsy syndrome of unknown etiology. Epilepsia 2009; 50: 49-51.

4 Ohba C, Kato M, Takahashi S, Lerman-Sagie T, Lev D, Terashima H, et al. Early onset epileptic encephalopathy caused by de novo SCN8A mutations. Epilepsia 2014; 55(7): 994-1000.

5 De Filippo MR, Rizzo F, Marchese G, Giurato G, Nassa G, Ravo M, et al. Lack of pathogenic mutations in six patients with MMPSI. Epilepsy Res 2014; 108(2): 340-344.

6 Burgess R, Wang S, McTague A, Boysen KE, Yang X, Zeng Q, et al. The Genetic Landscape of Epilepsy of Infancy with Migrating Focal Seizures. Ann Neurol 2019; 86(6): 821-831.

7 Rizzo F, Ambrosino P, Guacci A, Chetta M, Marchese G, Rocco T, et al. Characterization of two de novo KCNT1 mutations in children with malignant migrating partial seizures in infancy. Mol Cell Neurosci 2016; 72: 54-63.

8 Coppola G. Malignant migrating partial seizures in infancy. Handb Clin Neurol 2013; 111: 605-609.

9 Carranza RojoD, Hamiwka L, McMahon JM, Dibbens LM, Arsov T, Suls A, et al. De novo SCN1A mutations in migrating partial seizures of infancy. Neurology $2011 ; 77(4): 380-383$.

10 Shellhaas RA, Wusthoff CJ, Tsuchida TN, Glass HC, Chu CJ, Massey SL, et al. Profile of neonatal epilepsies: Characteristics of a prospective US cohort. Neurology 2017; 89(9): 893-899.

11 Olson HE, Kelly M, LaCoursiere CM, Pinsky R, Tambunan D, Shain C, et al. Genetics and genotype-phenotype correlations in early onset epileptic encephalopathy with burst suppression. Annals of neurology 2017; 81: 419-429.

12 McTague A, Howell KB, Cross JH, Kurian MA, Scheffer IE. The genetic landscape of the epileptic encephalopathies of infancy and childhood. Lancet Neurol 2016; 15: 304-316.

13 Mills PB, Surtees RA, Champion MP, Beesley CE, Dalton N, Scambler PJ, et al. Neonatal epileptic encephalopathy caused by mutations in the PNPO gene encoding pyridox(am)ine 5'-phosphate oxidase. Hum Mol Genet 2005; 14(8): 1077-1086.

14 Dibbens LM, Tarpey PS, Hynes K, Bayly MA, Scheffer IE, Smith R, et al. X-linked protocadherin 19 mutations cause female-limited epilepsy and cognitive impairment. Nat Genet 2008; 40(6): 776-781.

15 Mills PB, Struys E, Jakobs C, Plecko B, Baxter P, Baumgartner M, et al. Mutations in antiquitin in individuals with pyridoxine-dependent seizures. Nat Med 2006; 12(3): 307-309.

16 Miyamoto Y, Yamauchi J, Sanbe A, Tanoue A. Dock6, a Dock-C subfamily guanine nucleotide exchanger, has the dual specificity for Rac1 and Cdc42 and regulates neurite outgrowth. Exp Cell Res 2007; 313(4): 791-804.

17 Shaheen R, Faqeih E, Sunker A, Morsy H, Al-Sheddi T, Shamseldin HE, et al. Recessive mutations in DocK6, encoding the guanidine nucleotide exchange factor DOCK6, lead to abnormal actin cytoskeleton organization and Adams-Oliver syndrome. Am J Hum Genet 2011; 89(2): 328-333.

18 Ebrahimi-Fakhari D, Moufawad El AchkarC, Klein C. PRRT2-Associated Paroxysmal Movement Disorders 1993.

19 Vulpe C, Levinson B, Whitney S, Packman S, Gitschier J. Isolation of a candidate gene for Menkes disease and evidence that it encodes a coppertransporting ATPase. Nat Genet 1993; 3(1): 7-13.

20 Kaler SG, Gallo LK, Proud VK, Percy AK, Mark Y, Segal NA, et al. Occipital horn syndrome and a mild Menkes phenotype associated with splice site mutations at the MNK locus. Nat Genet 1994; 8(2): 195-202.

21 Kennerson ML, Nicholson GA, Kaler SG, Kowalski B, Mercer JF, Tang J, et al. Missense mutations in the copper transporter gene ATP7A cause X-linked distal hereditary motor neuropathy. Am J Hum Genet 2010; 86(3): 343-352.

22 Mallaret M, Synofzik M, Lee J, Sagum CA, Mahajnah M, Sharkia R, et al. The tumour suppressor gene WWOX is mutated in autosomal recessive cerebellar ataxia with epilepsy and mental retardation. Brain 2014; 137(Pt 2): 411-419.

Page $7 / 13$ 
23 Abdel-Salam G, Thoenes M, Afifi HH, Korber F, Swan D, Bolz HJ. The supposed tumor suppressor gene WWOX is mutated in an early lethal microcephaly syndrome with epilepsy, growth retardation and retinal degeneration. Orphanet J Rare Dis 2014; 9: 12.

24 Takahashi T, Honda, R, Nishikawa Y. Cloning of the human cDNA which can complement the defect of the yeast mannosyltransferase I-deficient mutant alg 1. Glycobiology 2000; 10(3): 321-327.

25 Schwarz M, Thiel C, Lubbehusen J, Dorland B, de Koning T, von Figura K. Deficiency of GDP-Man:GlcNAc2-PP-dolichol mannosyltransferase causes congenital disorder of glycosylation type Ik. Am J Hum Genet 2004. 74(3): 472-481.

26 Harshman LA, Ng BG, Freeze HH, Trapane P, Dolezal A, Brophy PD, et al. Congenital nephrotic syndrome in an infant with ALG1-congenital disorder of glycosylation. Pediatr Int 2016. 58(8): 785-788.

27 Rohlfing AK, Rust S, Reunert J, Tirre M, Du Chesne I, Wemhoff S, et al. ALG1-CDG: a new case with early fatal outcome. Gene 2014; 534(2): 345-351.

28 Caraballo R, Pasteris MC, Fortini PS, Portuondo E. Epilepsy of infancy with migrating focal seizures: six patients treated with bromide. Seizure 2014; 23(10): 899-902.

29 Mikati MA, Jiang YH, Carboni M, Shashi V, Petrovski S, Spillmann R, et al. Quinidine in the treatment of KCNT1-positive epilepsies. Ann Neurol 2015; 78(6): 995-999.

30 Marsh E, Melamed SE, Barron T, Clancy RR. Migrating partial seizures in infancy: expanding the phenotype of a rare seizure syndrome. Epilepsia 2005; 46: 568-572.

31 Yang H, Yin F, Gan S, Pan Z, Xiao T, Kessi M, et al. The Study of Genetic Susceptibility and Mitochondrial Dysfunction in Mesial Temporal Lobe Epilepsy. Mol Neurobiol 2020; 57(9): 3920-3930.

32 Lemke JR, Riesch E, Scheurenbrand T, Schubach M, Wilhelm C, Steiner I, et al. Targeted next generation sequencing as a diagnostic tool in epileptic disorders. Epilepsia 2012; 53(8): 1387-1398.

\section{Tables}

Table 1 Clinical features of the 36 children with MMPSI 


\begin{tabular}{|c|c|}
\hline Feature & Incidence \\
\hline Males: females & $25: 11 \rrbracket 2.3 \rrbracket$ \\
\hline Age 4 hours to 3 days at onset & $11 / 36(30.6 \%)$ \\
\hline Age $\leqq 6$ months at onset & $31 / 36(86.1 \%)$ \\
\hline $\begin{array}{l}\text { Family history of epilepsy, febrile seizure or mental retardation, stillbirth } \\
\text { Combined diseases }\end{array}$ & $10 / 36(27.8 \%)$ \\
\hline \multicolumn{2}{|l|}{ Congenital disorder of glycosylation, type Ik } \\
\hline Menkes disease & $1 / 36(2.8 \%)$ \\
\hline Intracranial infection & $1 / 36(2.8 \%)$ \\
\hline Inguinal hernia & $3 / 36(8.3 \%)$ \\
\hline Intracranial hemorrhage & $1 / 36(2.8 \%)$ \\
\hline Premature & $1 / 36(2.8 \%)$ \\
\hline Congenital heart disease (open foramen ovale, patent ductus arteriosus) & $1 / 36(2.8 \%)$ \\
\hline Perinatal history ( pneumonia, HIE, intrauterine distress $\rrbracket$ & $10 / 36(27.8 \%)$ \\
\hline \multicolumn{2}{|l|}{ Other (visual disturbance, pachygyria) } \\
\hline & $8 / 36(22.2 \%)$ \\
\hline & $2 / 36(5.6 \%)$ \\
\hline \multicolumn{2}{|l|}{ Seizure type } \\
\hline Focal & $36 / 36(100.0 \%)$ \\
\hline Tonic & $21 / 36(58.3 \%)$ \\
\hline Spasms & $8 / 36(22.2 \%)$ \\
\hline Clonic & $5 / 36(13.9 \%)$ \\
\hline Others(tonic-clonic, autonomic nerve, etc) & $8 / 36(22.2 \%)$ \\
\hline \multicolumn{2}{|l|}{ EEG findings } \\
\hline Seizure migration & $36 / 36(100.0 \%)$ \\
\hline Hypsarrhythmia & $13 / 36(36.1 \%)$ \\
\hline Burst suppression & $7 / 36(19.4 \%)$ \\
\hline \multicolumn{2}{|l|}{ Neuroimaging abnormal } \\
\hline Cerebral atrophy & $3 / 36(8.3 \%)$ \\
\hline Forehead dysplasia / Widened brain space & 4/36(11.1\%) \\
\hline Hippocampal sclerosis & $3 / 36(8.3 \%)$ \\
\hline Brain softening foci & $3 / 36(8.3 \%)$ \\
\hline Subependymal cyst & $5 / 36(13.9 \%)$ \\
\hline Dysplasia of the corpus callosum & $1 / 36(2.8 \%)$ \\
\hline Pachygyria & $1 / 36(2.8 \%)$ \\
\hline \multicolumn{2}{|l|}{ Genetic tests } \\
\hline Chromosome karyotype analysis & $1 / 36(2.8 \%)$ \\
\hline Copy number variant analysis & $1 / 36(2.8 \%)$ \\
\hline Mitochondrial genome sequencing & $3 / 36(8.3 \%)$ \\
\hline Epilepsy genes panel & $11 / 36(30.6 \%)$ \\
\hline Whole exome sequencing & $15 / 36(41.7 \%)$ \\
\hline No genetic tests & $10 / 36(27.8 \%)$ \\
\hline \multicolumn{2}{|l|}{ Seizure control } \\
\hline Seizure free & $14 / 36(38.9 \%)$ \\
\hline
\end{tabular}

Page 9/13 


\begin{tabular}{|c|c|}
\hline Reduced $>50 \%$ & $9 / 36(25.0 \%)$ \\
\hline Ineffective & $13 / 36(36.1 \%)$ \\
\hline \multicolumn{2}{|l|}{ Outcomes } \\
\hline Severe retardation & $14 / 36(38.9 \%)$ \\
\hline Mild-moderate retardation & $13 / 36(36.1 \%)$ \\
\hline Normal & $3 / 36(8.3 \%)$ \\
\hline Death & $6 / 36(16.7 \%)$ \\
\hline
\end{tabular}

Abbreviations: HIE= Hypoxic-ischemic encephalopathy; MMPSI=Malignant migrating partial seizures of infancy

Table 2 Variants in novel MMPSI genes and in silico analysis of pathogenicity

\begin{tabular}{|c|c|c|c|c|c|c|c|}
\hline Patient & Gene & Inheritance & gDNA(GRCh37/hg19) & Amino acid & rs ID & ExAc and gnomAD & $\begin{array}{l}\text { Poly- } \\
\text { phen2 }\end{array}$ \\
\hline 1 & KCNT1 & De Novo & Chr9:138651532G>A & p.G288S & rs587777264 & $0 ; 0$ & PD \\
\hline 2 & KCNT1 & De Novo & Chr9:138660694G>A & p.R474H & rs397515404 & $0 ; 0$ & PD \\
\hline 3 & KCNT1 & De Novo & Chr9:138670635C>T & p.A899V & - & $0 ; 0$ & PD \\
\hline 4 & $S C N 2 A$ & Maternal & Chr2:166246069G>A & p.R1918H & rs201718767 & $0.00004957203 ; 0.000129241$ & B \\
\hline 5 & $S C N 2 A$ & De Novo & Chr2:166237654G>A & p.A1500T & - & $0 ; 0$ & PD \\
\hline 6 & $S C N 2 A$ & De Novo & Chr2:166237657A>G & p.M1501V & - & $0 ; 0$ & PD \\
\hline \multirow[t]{2}{*}{7} & $A L D H 7 A 1 \#$ & Paternal & Chr5:125889987G>T & p.G398W & rs1347421419 & $0 ; 0$ & PD \\
\hline & & Maternal & Chr5:125918644dupA & p.I139fs & - & $0 ; 0$ & NA \\
\hline \multirow[t]{2}{*}{8} & PNPO & Paternal & Chr17:46022062G>A & p.S115N & - & $0 ; 0$ & PD \\
\hline & & Maternal & Chr17:46023290C>T & p.R161C & rs146027425 & $0 ; 0.00002473207$ & PD \\
\hline 9 & SCN1A & De Novo & Chr2:166909430T>C & p.L209P & NA & $0 ; 0$ & PD \\
\hline \multirow[t]{3}{*}{10} & WWOX & Maternal & Chr16:78133677T >C & p.M1? & rs758588684 & $0 ; 0.0000323039$ & PD \\
\hline & WWOX & Paternal & Chr16:78148874C>T & p.H78Y & - & $0 ; 0$ & PD \\
\hline & ATP7A\# & Maternal & ChrX:77271345DelG & p.G865Dfs*5 & - & $0 ; 0$ & PD \\
\hline \multirow[t]{2}{*}{11} & DOCK6\# & Maternal & Chr19:11324989T>C & p.Q1434X & rs1194206302 rs868514448 & $0 ; 0$ & NA \\
\hline & DOCK6\# & Paternal & Chr19:11354308G>A & p.A395T & & $0 ; 0.00000822145$ & PD \\
\hline 12 & PRRT2\# & Paternal & Chr16:29825016DupC & p.R217Pfs*8 & rs772994486 & $0 ; 0$ & NA \\
\hline 13 & PCDH19\# & De Novo & ChrX:99662806G>C & p.D264H & - & $0 ; 0$ & PD \\
\hline \multirow[t]{2}{*}{14} & ALG1 & Maternal & Chr16:5123195C>A & p.Q110K & rs774489344 & $0 ; 0$ & PD \\
\hline & & Paternal & Chr16:5129065A>G & NA & rs768733117 & $0.000017 ; 0$ & NA \\
\hline 15 & $S C N 2 A$ & De Novo & Chr2:166164381 & p.C137Y & - & $0 ; 0$ & PD \\
\hline 16 & $S C N 2 A$ & De Novo & Chr2:166170520 & p.G429L & rs1553568987 & - & PD \\
\hline 17 & $S C N 2 A$ & De Novo & Chr2:166165304 & p.A202V & - & - & PD \\
\hline
\end{tabular}

Abbreviations: $\mathrm{ACMG}=$ American College of Medical Genetics; $\mathrm{B}=$ Benign; $\mathrm{CMA}$ = clinical microarray; CNV=Copy Number Variant; $\mathrm{D}=$ Deleterious; ExAC = Exome Aggregation Consortium; gnomAD = Genome Aggregation Database; $L P=$ likely pathogenic; MMPSI=Malignant migrating partial seizures of infancy; $\mathrm{NA}=$ Not applicable: when the gene mutation is nonsense or frameshift mutation, the software can not predict the pathogenicity of the mutation; $\mathrm{P}=$ pathogenic; PD = Possibly Damaging; SIFT = Sorting Intolerant from Tolerant; $T$ = Tolerated; VUS = variant of unknown significance; WES = Whole Exome Sequencing; \#: novel gene

Table 3 Phenotype-genotype data of 36 patients with MMPSI 


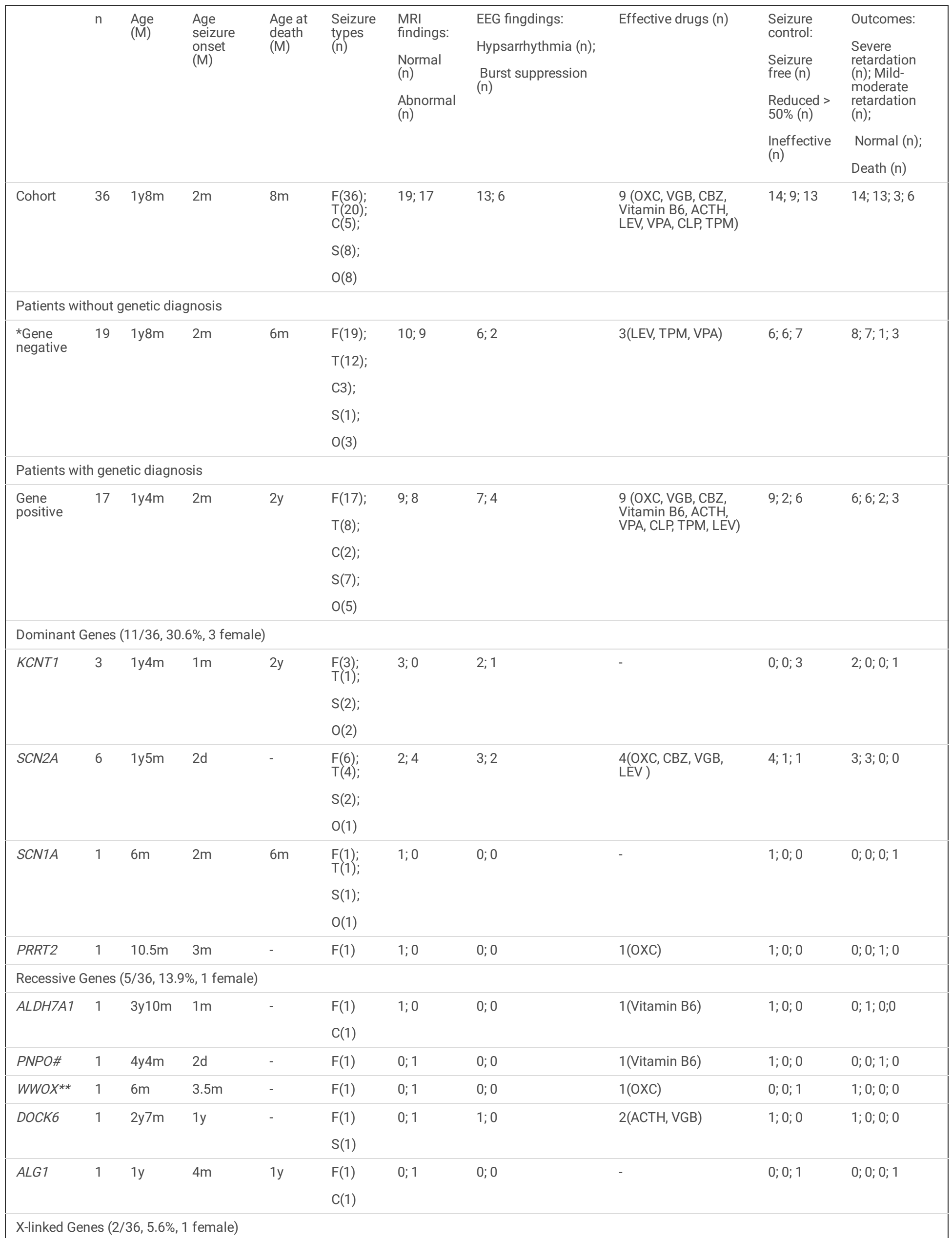




\begin{tabular}{|c|c|c|c|c|c|c|c|c|c|c|}
\hline$A T P 7 A^{* *}$ & 1 & $6 m$ & $3.5 \mathrm{~m}$ & - & $F(1)$ & $0 ; 1$ & $0 ; 0$ & $1(\mathrm{OXC})$ & $0 ; 0 ; 1$ & $1 ; 0 ; 0 ; 0$ \\
\hline \multirow[t]{3}{*}{ PCDH19\# } & 1 & $2 \mathrm{y} 11 \mathrm{~m}$ & $9 m$ & - & $F(1)$; & $1 ; 0$ & $1 ; 0$ & 3(TPM, VPA, CLB) & $0 ; 1 ; 0$ & $0 ; 1 ; 0 ; 0$ \\
\hline & & & & & $\mathrm{T}(1)$; & & & & & \\
\hline & & & & & $S(1)$ & & & & & \\
\hline
\end{tabular}

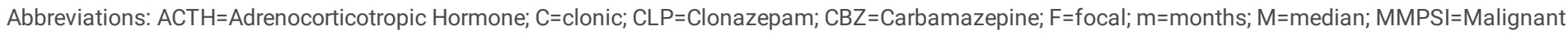

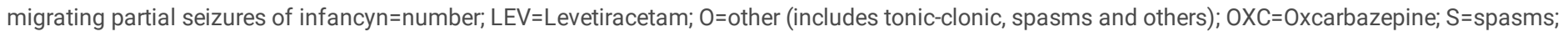
T=tonic; TPM=Topiramate; VGB=Vigabatrin; VPA=Valproic Acid; y=year; *: Gene negative patients include those who have had a genetic test and the result was negative and those who have not been tested; $\star *$ : one patient has two genetic mutations.; \#: The mutant was female.

Table 4 The table of MRI and prognosis

\begin{tabular}{|lll|}
\hline MRI & Seizure control: & Outcomes: \\
& Seizure free $(\%) ;$ & Normal (\%); \\
& Reduced $>50 \%(\%) ;$ & Mild-moderate retardation (\%); \\
& Ineffective (\%) & Severe retardation (\%); \\
& & Death (\%) \\
Normal & $9 / 19(47.4 \%)$ & $2 / 19(10.5 \%)$ \\
& $1 / 19(5.3 \%)$ & $8 / 19(42.1 \%)$ \\
& $9 / 19(47.4 \%)$ & $7 / 19(36.8 \%)$ \\
& & $2 / 19(10.5 \%)$ \\
\hline Abnormal & $5 / 17(29.4 \%)$ & $1 / 17(5.9 \%)$ \\
& $4 / 17(23.5 \%)$ & $5 / 17(29.4 \%)$ \\
& $8 / 17(47.1 \%)$ & $7 / 17(41.2 \%)$ \\
& & $4 / 17(23.5 \%)$ \\
\hline
\end{tabular}

Abbreviations: MRI=Magnetic resonance imaging

Table 5 The table of EEG and prognosis 


\begin{tabular}{|lll|}
\hline EEG & Seizure control: & Outcomes: \\
& Seizure free $(\%) ;$ & Normal (\%); \\
& Reduced $>50 \%(\%) ;$ & Mild-moderate retardation (\%); \\
& Ineffective $(\%)$ & Severe retardation (\%); \\
& & Death (\%) \\
\hline Hypsarrhythmia & $5 / 13(38.5 \%) ;$ & $0 / 13(0) ;$ \\
& $2 / 13(15.4 \%) ;$ & $5 / 13(38.5 \%) ;$ \\
& $6 / 13(46.1 \%)$ & $6 / 13(46.1 \%) ;$ \\
& & $2 / 13(15.4 \%)$ \\
\hline Burst suppression & $1 / 7(14.3 \%) ;$ & $0 / 7(0) ;$ \\
& $5 / 7(71.4 \%) ;$ & $1 / 7(14.3 \%) ;$ \\
& $1 / 7(14.3 \%)$ & $3 / 7(42.9 \%) ;$ \\
& & $3 / 7(42.9 \%)$ \\
\hline Hypsarrhythmia and burst suppression & $1 / 3(33.3 \%) ;$ & $0 / 3(0) ;$ \\
& $1 / 3(33.3 \%) ;$ & $1 / 3(33.3 \%) ;$ \\
& $1 / 3(33.3 \%)$ & $2 / 3(66.7 \%) ;$ \\
& & $0 / 3(0)$ \\
\hline & $8 / 19(42.1 \%) ;$ & $3 / 19(15.8 \%) ;$ \\
& $4 / 19(21.1 \%) ;$ & $9 / 19(47.4 \%) ;$ \\
& $7 / 19(36.8 \%)$ & $5 / 19(26.3 \%) ;$ \\
& & $2 / 19(10.5 \%)$ \\
\hline
\end{tabular}

Abbreviations: $\mathrm{EEG}=$ Electroencephalogram 